American Journal of Applied Sciences 4 (12): 1063-1070, 2007

ISSN 1546-9239

(C) 2007 Science Publications

\title{
Assessment of Soil Solution Chemicals after Tannery Effluents Disposal
}

\author{
${ }^{1}$ Célia A. Surita, ${ }^{1,2}$ Thomas Gloaguen, ${ }^{1,3}$ Célia R. Montes and ${ }^{4}$ Carlos T. S. Dias \\ ${ }^{1}$ Núcleo de Pesquisas em Geoquímica e Geofísica da Litosfera (Nupegel), Universidade de São Paulo. \\ Av. Pádua Dias, 11, 13418-900, Piracicaba, São Paulo, Brazil. \\ ${ }^{2}$ Instituto de Geociências, Universidade de São Paulo. Rua do Lago, 562, 05508-900, São Paulo, Brazil. \\ ${ }^{3}$ Departamento de Solos e Nutrição de Plantas, Universidade de São Paulo. Av. Pádua Dias, 11, 13418- \\ 900, Piracicaba, São Paulo, Brazil. \\ ${ }^{4}$ Departamento de Ciências Exatas, Universidade de São Paulo. Av. Pádua Dias, 11, 13418-900, \\ Piracicaba, São Paulo, Brazil
}

\begin{abstract}
Knowledge about soil solution chemicals is important for assessing their mobility, availability, migration to groundwater and toxicity to plants. The objective of this study was to apply factor analysis to data obtained on soil solution chemicals during a one-year monitoring program in a controlled experiment with tannery effluents disposed on the soil surface, to extract information on their relationship and identify the main contaminants. Seventeen chemical parameters were monitored at six different depths on soil profile, focusing on metals and nitrate in soil solution. Four Factors accounted for $79.20 \%$ of the total variance, of which the most important were: Factor $1(48.35 \%)$ showed significant loadings for $\mathrm{Mn}^{2+}, \mathrm{Na}^{+}, \mathrm{K}^{+}, \mathrm{Ca}^{2+}, \mathrm{Mg}^{2+}, \mathrm{Cl}^{-}, \mathrm{Pb}^{2+}$ and electric conductivity, strongly influenced by high load effluent disposal; Factor $2(12.21 \%)$ was related with $\mathrm{SO}_{4}{ }^{2+}$, Factor $3(10.16 \%)$ associated with $\mathrm{Cu}^{2+}$ and $\mathrm{Zn}^{2+}$ and Factor $4(8.49 \%)$ associated with nitrogen mineralization dynamics after high disposal.
\end{abstract}

Key words: Soil solution, groundwater, tannery effluents, factor analysis

\section{INTRODUCTION}

Industrial activities are capable of generating soil and groundwater pollution as a result of the emission of liquid effluents or waste disposal practices ${ }^{[1-5]}$. The tanning industry is considered an activity with elevated potential for environmental pollution all over the world. Tanning processes use substantial amounts of chromium salts and other heavy metals, sulfide and organic compounds ${ }^{[6]}$. Brazilian bovine hide tanneries usually consume 20 to $40 \mathrm{~m}^{3}$ of water per ton of processed hide ${ }^{[7]}$. According to IBGE $^{[8]}$, it is estimated that about 35 million hides from chrome processing were tanned in 2005, generating 24 to 49 million $\mathrm{m}^{3}$ of wastewater and 0.6 to 1.2 million tons of sludge.

A great deal of these effluents has been continuously discharged in soils in the northeast region of the State of São Paulo (SE, Brazil), affecting the soil and groundwater quality, but the effects of these practices are not yet well known. These soils are mineral soils formed under tropical climates subjected to intense weathering. They have a sandy clay loam to sandy loam texture, low activity clay, mainly kaolinite and low organic matter content ${ }^{[\rho]}$.

It is known that heavy metals added to soils are rapidly and specifically adsorbed by the solid fraction. However their availability, potential toxicity and mobility within the soil profile will depend upon the binding forms with clays, organic matter and hydrous oxides, oxides and oxyhydroxides; the interactions of their associations with time; the saturation of specific sites of adsorption; the crystallinity and morphology of absorbent surfaces; $\mathrm{pH}$ variation ${ }^{[10-13]}$ and physicochemical characteristics ${ }^{[14]}$.

Several studies have shown that the availability of $\mathrm{Cr}$ (III) in the soil solution is limited by the formation of hydroxides as $\mathrm{Cr}(\mathrm{OH})_{3}$ and $\mathrm{Cr}_{2} \mathrm{O}_{3}\left(\mathrm{H}_{2} \mathrm{O}\right)$, at pHs between 6 and $12^{[15]}$, or by co-precipitation with $\mathrm{Fe}$, forming $\quad\left(\mathrm{Cr}_{\mathrm{x}}, \mathrm{Fe}_{1-\mathrm{x}}\right)(\mathrm{OH})_{3}, \quad\left(\mathrm{Cr}_{\mathrm{x}}, \mathrm{Fe}_{1-\mathrm{x}}\right) \mathrm{OOH}$, $\mathrm{Fe}_{\mathrm{x}}, \mathrm{Cr}_{2 \mathrm{x}} \mathrm{O}_{3}{ }^{[16,17]}$. Another mechanism controlling $\mathrm{Cr}$ availability is the adsorption on the surface of $\mathrm{Fe}, \mathrm{Mn}$ and $\mathrm{Al}$ oxides and oxi-hydroxides and clay-minerals, at $\mathrm{pH}<6^{[18,19]}$ and adsorption onto organic matter ${ }^{[20]}$. The $\mathrm{Cr}$ (III) oxidation seems to be mainly controlled by the

Corresponding Author: Célia Alves Surita - Núcleo de Pesquisas em Geofísica e Geoquímica da Litosfera. Av. Pádua Dias, 11, 13418-900, Piracicaba, São Paulo, Brazil 
sorption on Mn-oxides surfaces followed by the electron transfer and desorption of $\mathrm{Cr}(\mathrm{VI})$ and $\mathrm{Mn}^{2+[21-}$ 23] and by $\mathrm{MnO}_{2}$ amount ${ }^{[24]}$. Tzou et al. ${ }^{[25]}$ showed that chromium oxidation by Mn-oxides was rapid at acidic conditions and kinetically slow at high $\mathrm{pH}$, inhibited by organic ligands.

Conservative solutes move with soil water and in response to solute concentration gradients ${ }^{[26]}$ and are influenced by soil hydraulic ${ }^{[27]}$ and heterogeneity ${ }^{[28]}$. These studies require monitoring of a wide range of physical, chemical and biological data. Multivariate analysis is a mathematical tool that can be employed to study the interrelationship among wide data sets by reducing the dimensionality of the data variables ${ }^{[29-31]}$.

Our interest in this work focuses on metal and nitrate levels in soil solution at different depths in an acid soil (Typic Haplustox) with tannery effluents disposal. The data obtained during a one-year monitoring program in a controlled experiment were subjected to factor analysis, to extract information on the relationship between soil solution chemicals and to identify the principal contaminants.

\section{MATERIALS AND METHODS}

The experiment took place in a $9 \mathrm{~m}^{2}$-experimental plot, located in Monte Aprazível (NW of the State of São Paulo, Brazil, $20^{\circ} 46^{\prime} \mathrm{S}, 49^{\circ} 42^{\prime} \mathrm{W}$ ), in the Aw climate zone, according to the Köppen classification. During the experiment (1996-1997) the annual average temperature was $25{ }^{\circ} \mathrm{C}$ and the annual average precipitation was $1400 \mathrm{~mm}$. The driest period was observed between the months of July and August/97 (0 $\mathrm{mm})$. The period with most rainfall was from November/96 to March/97 with precipitations from 118 $\mathrm{mm}$ to $321 \mathrm{~mm}$.

In the experimental plot, 6 pressure-vacuum lysimeters with ceramic porous cups were installed at $0.5 \mathrm{~m}$ intervals to $3.0 \mathrm{~m}$ of depth to sample soil solution according to ASTM procedures ${ }^{[32]}$. The sampling was done applying a continuous suction of $20-40$ to $60 \mathrm{kPa}$ per period of 10 to 12 hours. Soil and soil solution samplings were first performed before the disposal of effluents, at the same depths. The effluents were applied to the soil in two amounts and periods: $700 \mathrm{~L}$ in March/1996 and 1,700 L in September/1996. The tannery effluents were collected during a whole working day and were analyzed for the determination of $\mathrm{pH}$, electrical conductivity (EC), $\mathrm{Cr}_{\text {total }}, \mathrm{Mn}, \mathrm{Fe}_{\text {total }}, \mathrm{Al}^{3+}$, $\mathrm{Zn}^{2+}, \mathrm{Cu}^{2+}, \mathrm{Ni}^{2+}, \mathrm{Pb}^{2+} \mathrm{Na}^{+}, \mathrm{K}^{+}, \mathrm{Ca}^{2+}, \mathrm{Mg}^{2+}, \mathrm{S}^{2-}, \mathrm{SO}_{4}{ }^{2-}$, $\mathrm{Cl}^{-}, \mathrm{NH}_{3}, \mathrm{NO}_{3}^{-}$, chemical oxygen demand (COD), biological oxygen demand (BOD), settleable solids (SetS) and suspended solids (SS) (Table 1). Metals were determined by atomic absorption spectrometer, anions by spectrophotometry UV/VIS, COD by acidic chromate solution, BOD by Azide Modified Winkler Method, SS by filtration, setteable solids by Imhoff cone, by Standard Methods ${ }^{[33]}$.

Soil solution samples were collected in February/96, March/96 (before disposal), May/96, June/96, July/96 (after first disposal), October/96, November/96, January/97 and March/97 (after second disposal); filtered through $0.45 \mu \mathrm{m}$ cellulose acetate and the preservation was conducted by Standard Methods $^{[33]}$. From every sample, a subsample was kept at its natural $\mathrm{pH}$ and used for determination of anions $\left(\mathrm{NO}_{3}{ }^{-}, \mathrm{SO}_{4}{ }^{2-}, \mathrm{Cl}^{-}\right.$and $\left.\mathrm{PO}_{4}{ }^{3-}\right)$ by liquid chromatography. A second subsample was acidified to $\mathrm{pH} 2$ with nitric acid for metal analysis $\left(\mathrm{Cr}_{\text {total }}, \mathrm{Fe}_{\text {total }}, \mathrm{Mn}^{2+}, \mathrm{Al}^{3+}, \mathrm{Zn}^{2+}\right.$, $\mathrm{Cu}^{2+}, \mathrm{Ni}^{2+}, \mathrm{Pb}^{2+}, \mathrm{Ca}^{2+}$ and $\mathrm{Mg}^{2+}$ ) by atomic absorption spectrophotometer. A third subsample was acidified to $\mathrm{pH} 2$ with sulphuric acid for $\mathrm{Na}^{+}$and $\mathrm{K}^{+}$analysis by flame atomic absorption spectroscopy. Analytical data was controlled by calibration done with standard solutions in the appropriate matrix and analyzed at the beginning of series and after 10 samples series. Besides the check samples, regularly repeated analyses of the same samples were done. Electric conductivity (EC) and $\mathrm{pH}$ measurements were performed in situ (Table 2).

The soil samples were stored at $4^{\circ} \mathrm{C}$, dried at $25^{\circ} \mathrm{C}$, homogenized, quartered and sieved at $2 \mathrm{~mm}$. They were analyzed for the determination of $\mathrm{pH}$ (electrode, $0.01 \mathrm{molL}^{-1} \mathrm{CaCl}_{2}$ ), organic matter, exchangeable ions and cation exchange capacity (cation exchange resin, $\left.1 \mathrm{~N} \mathrm{NaHCO}_{3}, \mathrm{pH} 8.5\right)^{[34]}$, texture ${ }^{[35]}$ and chemical composition (X-ray fluorescence) (Table 3).

Statistical analysis: The correlation coefficients were calculated for the 17 variables values that presented more than 6 valid cases, accepted to factor analysis. Factor analysis was performed from the correlation matrix to extract principal factors with eigenvalues greater than 1 and detect the relationship between the variables. The selected factors were subjected to normalized varimax rotation in order to define a clear pattern of loadings ${ }^{[36]}$. Analysis of variance was employed on the factor scores to evaluate the effects of effluent load and different depths on the extracted factors. The statistical analyses were carried out in Statistica software package ${ }^{[37]}$.

\section{RESULTS AND DISCUSSION}

The effluents discharged into the soil presented high variability of the chemical and physical characteristics due to different quantities of processed 
Am. J. Appl. Sci., 4 (12): 1063-1070, 2007

Table 1: Physical and chemical characteristics of effluents disposed into the soil

\begin{tabular}{|c|c|c|}
\hline Effluents parameters & $\begin{array}{r}\text { March-96 } \\
700 \mathrm{~L} \\
\end{array}$ & $\begin{array}{r}\text { September }-96 \\
1,700 \mathrm{~L} \\
\end{array}$ \\
\hline $\mathrm{pH}$ & 7.1 & 7.5 \\
\hline $\mathrm{EC}\left(\mathrm{dS} \mathrm{m}^{-1}\right)$ & 11.5 & 10.9 \\
\hline $\mathrm{Cr}_{\text {total }}\left(\mathrm{mg} \mathrm{L}^{-1}\right)$ & 138 & 41 \\
\hline $\mathrm{Fe}_{\text {total }}\left(\mathrm{mg} \mathrm{L}^{-1}\right)$ & 4.4 & 2.2 \\
\hline $\mathrm{Mn}^{2+}\left(\mathrm{mg} \mathrm{L}^{-1}\right)$ & 0.01 & 0.01 \\
\hline $\mathrm{Al}^{3+}\left(\mathrm{mg} \mathrm{L}^{-1}\right)$ & 11.5 & 25.5 \\
\hline $\mathrm{Ni}^{2+}\left(\mathrm{mg} \mathrm{L}^{-1}\right)$ & 1.1 & 0.4 \\
\hline $\mathrm{Cu}^{2+}\left(\mathrm{mg} \mathrm{L}^{-1}\right)$ & 0.35 & 0.05 \\
\hline $\mathrm{Zn}^{2+}\left(\mathrm{mg} \mathrm{L}^{-1}\right)$ & 1.7 & 0.6 \\
\hline $\mathrm{Pb}^{2+}\left(\mathrm{mg} \mathrm{L}^{-1}\right)$ & 0.02 & 0.1 \\
\hline $\mathrm{Na}^{+}\left(\mathrm{mg} \mathrm{L}^{-1}\right)$ & 27,400 & 5,800 \\
\hline $\mathrm{K}^{+}\left(\mathrm{mg} \mathrm{L}^{-1}\right)$ & 44 & 23 \\
\hline $\mathrm{Ca}^{2+}\left(\mathrm{mg} \mathrm{L}^{-1}\right)$ & 355 & 453 \\
\hline $\mathrm{Mg}^{2+}\left(\mathrm{mg} \mathrm{L}^{-1}\right)$ & 25 & 15 \\
\hline $\mathrm{S}^{2-}\left(\mathrm{mg} \mathrm{L}^{-1}\right)$ & 19 & 26 \\
\hline $\mathrm{SO}_{4}^{2-}\left(\mathrm{mg} \mathrm{L}^{-1}\right)$ & 4,661 & 576 \\
\hline $\mathrm{Cl}^{-}\left(\mathrm{mg} \mathrm{L}^{-1}\right)$ & 14,670 & 3,548 \\
\hline $\mathrm{NH}_{3}\left(\mathrm{mg} \mathrm{L}^{-1}\right)$ & ND & 1058 \\
\hline $\mathrm{NO}_{3}^{-}\left(\mathrm{mg} \mathrm{L}^{-1}\right)$ & 36.0 & 1.2 \\
\hline $\operatorname{COD}\left(\mathrm{mg} \mathrm{L}^{-1}\right)$ & 4,081 & 3,232 \\
\hline $\mathrm{BOD}\left(\mathrm{mg} \mathrm{L}^{-1}\right)$ & 957 & 1,395 \\
\hline Suspended solid $\left(\mathrm{mgL}^{-1}\right)$ & 4,420 & 780 \\
\hline Settable Solids $\left(\mathrm{mgL}^{-1}\right)$ & 66 & ND \\
\hline $\begin{array}{l}\text { Sodium adsorption ratio } \\
\qquad\left(\mathrm{mmol}_{\mathrm{c}} \mathrm{L}^{-1}\right)^{-0,5}\end{array}$ & 379 & 73 \\
\hline
\end{tabular}

EC: Electrical conductivity, ND: not determined, COD: chemical oxygen demand, BOD: biological oxygen demand

hides and different batches at different days. Higher concentration of $\mathrm{Na}^{+}\left(5,800-27,400 \mathrm{mgL}^{-1}\right)$ was observed comparing to $\mathrm{Ca}^{2+}\left(350-450 \mathrm{mgL}^{-1}\right), \mathrm{K}^{+}(23-44$ $\left.\mathrm{mgL}^{-1}\right)$ and $\mathrm{Mg}^{2+}\left(15-25 \mathrm{~mL}^{-1}\right)$ (Table 1). This reflects the use of large amounts of $\mathrm{NaCl}$ in the hide preservation stage and $\mathrm{Na}_{2} \mathrm{~S}, \mathrm{NaOH}$ and $\mathrm{Na}_{2} \mathrm{SO}_{3}$ used during the tanning process. Among the heavy metals, $\mathrm{Cr}_{\text {total }}$ was found in high concentration $\left(41-138 \mathrm{mgL}^{-1}\right)$ and low levels of $\mathrm{Fe}_{\text {total }}\left(2.2-4.4 \mathrm{mgL}^{-1}\right), \mathrm{Mn}^{2+}(0.01$ $\left.\mathrm{mgL}^{-1}\right), \mathrm{Ni}^{2+}\left(0.4-1.1 \mathrm{mgL}^{-1}\right), \mathrm{Zn}^{2+}\left(0.6-1.7 \mathrm{mgL}^{-1}\right)$ and $\mathrm{Pb}^{2+}\left(0.10-0.02 \mathrm{mgL}^{-1}\right)$. The $\mathrm{Cr}$ concentrations are much higher than the Brazilian standard for $\mathrm{Cr}$ bearing discharges in water bodies. The $\mathrm{Al}^{3+}$ presents levels between 11.5-25.5 mgL ${ }^{-1}$, due to the aluminum salts used before chromium tanning. The effluents present a neutral $\mathrm{pH}$ value due to $\mathrm{NaOH}, \mathrm{Ca}(\mathrm{OH})_{2}, \mathrm{Mg}(\mathrm{OH})_{2}$, $\mathrm{CaO}$, used during the initial stages of tanning.

The presence of $\mathrm{Fe}_{\text {total }}, \mathrm{Mn}^{2+}, \mathrm{Zn}^{2+}, \mathrm{Na}^{+}, \mathrm{K}^{+}, \mathrm{Ca}^{2+}$, $\mathrm{Mg}^{2+}, \mathrm{Cl}^{-}, \mathrm{NO}_{3}{ }^{-}$and $\mathrm{SO}_{4}{ }^{2-}$ in soil solution (Table 2), before effluent disposal, has been attributed to compositions of the total soil water, collected from different pore sizes, which have different mobilities ${ }^{[38]}$. The $\mathrm{pH}$ values of the soil solution (mean: 7.03) can reflect non equilibrium condition between soil and soil solution chemistry due to abundant rainfall (summer) that change the moisture content regulating the availability of the elements. Also, the sampling procedures of the soil solution, duration and degree of sampler vacuum, may change $\mathrm{pH}$ values ${ }^{[39]}$.

After the first disposal (700L), the $\mathrm{pH}$ decreased in the subsurface $\left(\begin{array}{ll}0.5 & \mathrm{~m}\end{array}\right)$ attributed to nitrogen transformations, which affect the acid-base chemistry of the soil and the soil solution. These conditions increased the solubility of $\mathrm{Al}^{3+}$ and $\mathrm{Mn}^{2+}$. Chromium was not observed in soil solution, probably due to the occurrence of reducing agents, such as ferrous iron and organic matter and sorption onto iron oxi-hydroxides that might contribute to the retention of chromium in trivalent state.

With the second disposal, it was observed that ion concentration increased up to approximately $2.0 \mathrm{~m}$ in depth indicating movement of $\mathrm{Na}^{+}, \mathrm{K}^{+}, \mathrm{Ca}^{2+}, \mathrm{Mg}^{2+}, \mathrm{Cl}^{-}$, $\mathrm{NO}_{3}{ }^{-}$and $\mathrm{Mn}^{2+}$. In spite of the great amount disposed $(1,700 \mathrm{~L})$, the concentration of $\mathrm{Cr}_{\text {total }}$ was below the detection limit of the analytical method. In some samples, iron concentration was below the detection limit, indicating that it may have contributed to retention of chromium in the trivalent state probably through co-precipitation reactions, reducing the chance of toxicity for plants and downward migration in the soil profile.

The correlation coefficient matrix is shown in Table 4. The highest correlation $(r=0.93 ; \mathrm{p}<0.01)$ occurred between $\mathrm{Ca}^{2+}$ and $\mathrm{Mg}^{2+}$ and $\mathrm{Mn}^{2+}$ versus $\mathrm{Ca}^{2+}$ and $\mathrm{Mg}^{2+}(\mathrm{r}=0.85, \mathrm{p}<0.01)$. Strong and negative correlations were obtained for $\mathrm{pH}$ versus $\mathrm{Mn}^{2+}(\mathrm{r}>-0.77$, $\mathrm{p}<0.01)$. The $\mathrm{Mn}^{2+}$ presented low concentrations in effluents, but the increase of its availability in soil solution (Table 2), might be related to Mn-oxide reduction caused by $\mathrm{Cr}(\mathrm{III})$ and $\mathrm{pH}$ decrease. The availability of $\mathrm{Al}^{3+}$ was attributed to $\mathrm{pH}$ decrease $(\mathrm{pH}<5.0)$, according to $\mathrm{McBride}^{[40]}$ and to higher potential acidity of these acid soil ${ }^{[41]} \cdot \mathrm{Pb}^{2+}$ also present a significant but lower correlation with $\mathrm{pH}(-0.48)$, pointing to heavy metal mobilization and acidification processes. The positive correlation of $\mathrm{NO}_{3}{ }^{-}$with $\mathrm{Ca}^{2+}$, $\mathrm{Mg}^{2+}, \mathrm{Mn}^{2+}$ and $\mathrm{Cl}^{-}(\mathrm{r}>0.40 \mathrm{p}<0.01)$ can be the result of a similar solubility and mobility through the unsaturated zone.

R-mode factor analysis: Eigenvalues indicated that the first four Factors accounted for $79.20 \%$ of the total variance, where all these eigenvalues are greater than one (Table 5). The first Factor accounted for $48.35 \%$ of the total variance and presented high loadings $(>0.7)$ for $\mathrm{EC}, \mathrm{Mn}^{2+}, \mathrm{Pb}^{2+}, \mathrm{Na}^{+}, \mathrm{K}^{+}, \mathrm{Ca}^{2+}, \mathrm{Mg}^{2+}$ and $\mathrm{Cl}^{-}$. This Factor, called "salinity", emphasizes the important role of $\mathrm{Na}^{+}, \mathrm{Mg}^{2+}, \mathrm{K}^{+}, \mathrm{Ca}^{2+}, \mathrm{Cl}^{-}, \mathrm{Mn}^{2+}$ and $\mathrm{Pb}^{2+}$ in the composition of soil solution, related to the effect of high load effluent disposal, with high salinity. 
Am. J. Appl. Sci., 4 (12): 1063-1070, 2007

Table 2: Soil solution quality attributes at different depths before and after effluent disposal

\begin{tabular}{|c|c|c|c|c|c|c|c|c|c|c|c|c|c|c|c|c|c|c|c|c|}
\hline & & Effluent & & $\mathrm{pH}$ & EC & $\mathrm{Cr}_{\text {total }}$ & $\mathrm{Fe}_{\text {total }}$ & $\mathrm{Mn}^{2+}$ & $\mathrm{Al}^{3+}$ & $\mathrm{Zn}^{2+}$ & $\mathrm{Cu}^{2+}$ & $\mathrm{Ni}^{2+}$ & $\mathrm{Pb}^{2+}$ & $\mathrm{Na}^{+}$ & $\mathrm{K}^{+}$ & $\mathrm{Ca}^{2+}$ & $\mathrm{Mg}^{2+}$ & $\mathrm{NO}_{3}{ }^{-}$ & $\mathrm{SO}_{4}{ }^{2-}$ & $\mathrm{Cl}^{-}$ \\
\hline & data & (L) & $(\mathrm{m})$ & & $\mathrm{dSm}^{-1}$ & \multicolumn{15}{|c|}{$\mathrm{mgL}^{-1}$} \\
\hline \multirow{12}{*}{$\begin{array}{l}\text { before } \\
\text { disposal }\end{array}$} & fev96 & 0 & 0.5 & 8.1 & 0.23 & BDL & 0.06 & BDL & BDL & 0.83 & 0.18 & BDL & BDL & 11.3 & 22.5 & 8.10 & 3.85 & 3.8 & 17 & 7.0 \\
\hline & fev96 & 0 & 1.0 & 7.6 & 0.16 & BDL & 0.06 & BDL & BDL & 0.59 & BDL & BDL & BDL & 7.0 & 20.0 & 5.15 & 2.65 & n.a & 5 & 4.7 \\
\hline & fev96 & 0 & 1.5 & 7.7 & 0.29 & BDL & $\mathrm{n} \cdot \mathrm{a}$ & 0.02 & BDL & 0.17 & 0.09 & BDL & BDL & 9.0 & 34.0 & 9.70 & 5.05 & 75.5 & 2 & 11.3 \\
\hline & fev96 & 0 & 2.0 & 6.9 & 0.31 & BDL & 0.10 & 0.04 & BDL & 0.66 & 0.27 & BDL & BDL & 10.0 & 12.0 & 16.15 & 8.35 & 85.7 & 4 & 20.5 \\
\hline & fev96 & 0 & 2.5 & 6.7 & 0.23 & BDL & 0.15 & 0.02 & BDL & 1.24 & 2.14 & BDL & BDL & 11.5 & 12.0 & 11.25 & 6.50 & 49.4 & 4 & 6.4 \\
\hline & fev96 & 0 & 3.0 & 6.6 & 0.20 & BDL & 0.07 & 0.04 & BDL & 1.11 & 2.62 & BDL & BDL & 3.8 & 8.8 & 7.65 & 5.00 & 29.2 & 7 & 4.1 \\
\hline & mar96 & 0 & 0.5 & 7.2 & 0.21 & BDL & 0.09 & 0.00 & BDL & 0.10 & BDL & BDL & BDL & 11.3 & 22.5 & 7.75 & 3.95 & 2.0 & 18 & 9.0 \\
\hline & mar96 & 0 & 1.0 & 7.1 & 0.14 & BDL & 0.05 & 0.02 & BDL & 0.21 & BDL & BDL & BDL & 7.0 & 20.0 & 7.90 & 3.85 & 4.0 & 2 & 7.0 \\
\hline & mar96 & 0 & 1.5 & 6.8 & 0.27 & BDL & 0.07 & 0.03 & BDL & 0.32 & BDL & BDL & 0.11 & 9.0 & 34.0 & 13.05 & 6.25 & 93.0 & 1 & 11.0 \\
\hline & mar96 & 0 & 2.0 & 6.5 & 0.39 & BDL & 0.16 & 0.04 & BDL & 0.22 & BDL & BDL & 0.08 & 10.0 & 12.0 & 26.00 & 12.00 & 145.0 & 2 & 6.0 \\
\hline & mar96 & 0 & 2.5 & 6.6 & 0.21 & BDL & 0.05 & 0.03 & BDL & 0.21 & BDL & BDL & BDL & 11.5 & 12.0 & 11.30 & 6.05 & 57.0 & 2 & 4.0 \\
\hline & mar96 & 0 & 3.0 & 6.6 & 0.17 & BDL & 0.07 & 0.02 & BDL & 0.11 & BDL & BDL & 0.17 & 3.8 & 8.8 & 10.25 & 7.95 & 51.0 & 3 & 4.0 \\
\hline \multirow{18}{*}{$\begin{array}{c}\text { after } \\
\text { first } \\
\text { disposal }\end{array}$} & may96 & 700 & 0.5 & 6.4 & 1.99 & BDL & 0.10 & 2.67 & $\mathrm{BDL}$ & 0.53 & BDL & 0.14 & 0.14 & 1380.4 & 102.5 & 170.00 & 49.00 & 528.0 & 98 & 70.0 \\
\hline & may96 & 700 & 1.0 & 7.4 & 0.12 & BDL & 0.15 & BDL & BDL & 0.14 & BDL & BDL & 0.09 & 3.9 & 10.2 & 7.55 & 2.65 & 28.6 & BDL & 6.0 \\
\hline & may96 & 700 & 1.5 & 7.0 & 0.26 & BDL & 0.37 & BDL & BDL & 0.07 & BDL & BDL & 0.07 & 6.1 & 24.1 & 10.85 & 5.00 & 114.4 & BDL & 8.5 \\
\hline & may96 & 700 & 2.0 & n.a & n.a & n.a & n.a & n.a & n.a & n.a & n.a & n.a & n.a & n.a & n.a & n.a & n.a & n.a & n.a & n.a \\
\hline & may96 & 700 & 2.5 & 6.6 & 0.20 & BDL & 0.21 & 0.02 & BDL & 0.11 & BDL & BDL & 0.09 & 10.4 & 11.9 & 9.40 & 6.55 & 88.0 & $\mathrm{n} . \mathrm{a}$ & 4.5 \\
\hline & may96 & 700 & 3.0 & 6.9 & 0.19 & n.a & n.a & n.a & n.a & n.a & n.a & BDL & n.a & n.a & n.a & n.a & n.a & 85.8 & 0 & 4.5 \\
\hline & jun96 & 700 & 0.5 & 5.5 & 3.65 & BDL & 0.24 & 1.40 & 0.80 & 0.97 & BDL & BDL & 0.06 & 676.1 & 33.8 & 43.00 & 13.50 & 327.8 & 713 & 410.0 \\
\hline & jun96 & 700 & 1.0 & 7.3 & 0.12 & BDL & 0.17 & BDL & BDL & 0.19 & BDL & BDL & 0.17 & 7.5 & 13.8 & 11.40 & 3.60 & 41.8 & 5 & 7.5 \\
\hline & jun96 & 700 & 1.5 & 8.0 & 0.27 & BDL & 0.08 & BDL & BDL & 0.18 & BDL & BDL & 0.06 & 10.0 & 37.5 & 11.00 & 4.80 & 103.4 & 5 & 11.0 \\
\hline & jun96 & 700 & 2.0 & 7.1 & 0.45 & BDL & 0.16 & 0.08 & BDL & 0.67 & BDL & BDL & 0.07 & 13.8 & 43.8 & 37.90 & 14.70 & 202.4 & 5 & 20.0 \\
\hline & jun96 & 700 & 2.5 & 6.3 & 0.19 & BDL & 0.21 & 0.10 & BDL & 0.16 & BDL & BDL & 0.08 & 16.3 & 12.5 & 16.10 & 9.00 & 85.8 & 5 & 20.0 \\
\hline & jun96 & 700 & 3.0 & 6.7 & 0.21 & n.a & n.a & n.a & n.a & n.a & n.a & n.a & n.a & n.a & $\mathrm{n} \cdot \mathrm{a}$ & n.a & n.a & n.a & n.a & 28.0 \\
\hline & july96 & 700 & 0.5 & 4.1 & 1.99 & BDL & 0.32 & 1.91 & 6.90 & 1.51 & BDL & 0.04 & 0.23 & n.a & n.a & 50.40 & 13.20 & 127.6 & n.a & n.a \\
\hline & july96 & 700 & 1.0 & 7.1 & 0.10 & BDL & 0.36 & BDL & BDL & 0.19 & BDL & BDL & 0.11 & 8.0 & 17.5 & 5.30 & 1.80 & 187.0 & BDL & 17.5 \\
\hline & july96 & 700 & 1.5 & 6.8 & 0.25 & BDL & 0.06 & BDL & BDL & 0.05 & BDL & BDL & BDL & 5.7 & 34.5 & 11.30 & 4.60 & 90.2 & 1 & 14.5 \\
\hline & july96 & 700 & 2.0 & 6.7 & 0.43 & BDL & 0.11 & 0.06 & BDL & 0.19 & BDL & BDL & BDL & 4.1 & 39.5 & 32.00 & 13.20 & 204.6 & BDL & 22.5 \\
\hline & july96 & 700 & 2.5 & 6.3 & 0.17 & BDL & 0.28 & 0.02 & BDL & 0.15 & BDL & BDL & BDL & 7.6 & 9.1 & 6.10 & 5.30 & 61.6 & 1 & 12.5 \\
\hline & july96 & 700 & 3.0 & 6.2 & 0.24 & 0.05 & 0.12 & 0.04 & BDL & 0.41 & BDL & BDL & BDL & 5.5 & 9.9 & 17.20 & 9.80 & 75.5 & BDL & 11.0 \\
\hline \multirow{20}{*}{$\begin{array}{c}\text { after } \\
\text { second } \\
\text { disposal }\end{array}$} & oct96 & 1700 & 0.5 & 7.1 & 0.63 & 0.06 & 0.26 & 0.66 & BDL & 1.57 & BDL & 0.07 & 0.10 & 1010.0 & 10.0 & 85.50 & 16.60 & 1782.0 & 762 & 584.2 \\
\hline & oct96 & 1700 & 1.0 & 6.1 & 1.18 & BDL & 0.24 & 3.93 & 1.80 & 0.64 & BDL & 0.10 & 0.17 & 1090.0 & 390.0 & 218.00 & 112.50 & 38.7 & 2116 & 2745.3 \\
\hline & oct96 & 1700 & 1.5 & 5.4 & 1.06 & BDL & 0.12 & 4.70 & BDL & 0.55 & 0.03 & 0.09 & 0.34 & 1640.0 & 190.0 & 150.00 & 85.50 & 44.0 & 13 & 3565.7 \\
\hline & oct96 & 1700 & 2.0 & 6.0 & 0.43 & BDL & 0.09 & 1.80 & n.a & 0.56 & BDL & 0.08 & 0.15 & 600.0 & 70.0 & 161.50 & 49.00 & 671.0 & 2 & 889.4 \\
\hline & oct96 & 1700 & 2.5 & 6.1 & 0.36 & BDL & 0.15 & 0.06 & BDL & 0.36 & BDL & BDL & 0.06 & 24.0 & 14.5 & 14.35 & 12.60 & 165.0 & 2 & 19.2 \\
\hline & oct96 & 1700 & 3.0 & 6.7 & 0.25 & BDL & 0.06 & 0.04 & BDL & 0.67 & BDL & BDL & 0.17 & 8.4 & 4.2 & 14.35 & 9.95 & 68.2 & 3 & 7.1 \\
\hline & nov96 & 1700 & 0.5 & 4.9 & 2.77 & 0.14 & 0.41 & 0.78 & 1.54 & 0.55 & BDL & 0.04 & 0.06 & 335.0 & 6.7 & 63.00 & 8.50 & 396.0 & 50 & 50.0 \\
\hline & nov96 & 1700 & 1.0 & 5.9 & 7.55 & BDL & 0.07 & 1.50 & BDL & 0.28 & BDL & 0.05 & 0.08 & 1420.0 & 60.0 & 92.00 & 34.75 & 2346.7 & 1050 & 300.0 \\
\hline & nov96 & 1700 & 1.5 & 4.7 & 11.16 & BDL & 0.09 & 5.70 & 3.95 & 0.14 & 0.03 & 0.09 & 0.18 & 1940.0 & 100.0 & 130.00 & 77.50 & 220.0 & BDL & 1500.0 \\
\hline & nov96 & 1700 & 2.0 & 4.9 & 6.85 & BDL & BDL & 1.40 & 2.15 & 0.48 & BDL & 0.07 & 0.16 & 2000.0 & 180.0 & 126.50 & 47.80 & 1122.0 & BDL & 983.3 \\
\hline & nov96 & 1700 & 2.5 & 5.7 & 0.54 & BDL & BDL & 0.02 & BDL & 0.22 & BDL & BDL & BDL & 55.0 & 15.0 & 9.80 & 12.15 & 187.0 & BDL & 375.0 \\
\hline & nov96 & 1700 & 3.0 & 6.0 & 0.25 & BDL & 0.05 & 0.03 & BDL & 0.49 & 0.03 & BDL & 0.30 & 9.0 & 11.0 & 11.40 & 11.90 & 88.0 & 20 & 5.0 \\
\hline & jan97 & 1700 & 0.5 & 6.3 & 0.16 & BDL & BDL & 0.02 & BDL & 0.09 & BDL & BDL & BDL & 10.0 & 0.9 & 4.12 & 0.44 & 5.5 & 8 & 1.4 \\
\hline & jan97 & 1700 & 1.0 & 6.2 & 1.06 & BDL & BDL & 0.09 & BDL & 0.12 & BDL & BDL & BDL & 179.6 & 17.5 & 8.50 & 3.55 & 50.6 & 55 & n.a \\
\hline & jan97 & 1700 & 1.5 & 5.4 & 5.48 & BDL & BDL & 1.80 & 0.58 & 0.18 & BDL & 0.04 & 0.18 & 1156.8 & 42.5 & 105.50 & 20.25 & 1424.5 & 205 & 497.0 \\
\hline & jan97 & 1700 & 2.0 & 4.7 & 10.17 & BDL & 0.07 & 7.46 & 5.50 & 0.44 & 0.04 & 0.11 & 0.33 & 1874.8 & 100.0 & 156.00 & 86.50 & 0.8 & BDL & 1830.0 \\
\hline & jan97 & 1700 & 2.5 & 5.4 & 8.24 & BDL & BDL & 2.06 & 0.85 & 0.49 & BDL & 0.07 & 0.25 & 1645.4 & 60.0 & 240.63 & 118.75 & 3.5 & BDL & 1300.0 \\
\hline & jan97 & 1700 & 3.0 & 5.3 & 6.04 & BDL & BDL & 0.03 & 2.30 & 1.04 & 0.03 & 0.12 & 0.33 & 638.3 & 40.0 & 31.63 & 33.13 & 5.3 & 12 & 800.0 \\
\hline & mar97 & 1700 & 0.5 & 6.0 & 0.09 & BDL & BDL & 0.03 & BDL & 0.21 & BDL & BDL & BDL & 9.0 & 6.9 & 4.20 & 1.30 & 60.7 & BDL & 0.5 \\
\hline & mar97 & 1700 & 1.0 & 6.5 & 0.71 & BDL & 0.05 & 0.02 & BDL & 0.17 & BDL & BDL & BDL & 99.7 & 10.0 & 5.20 & 2.60 & 82.7 & 263 & 11.5 \\
\hline
\end{tabular}


Am. J. Appl. Sci., 4 (12): 1063-1070, 2007

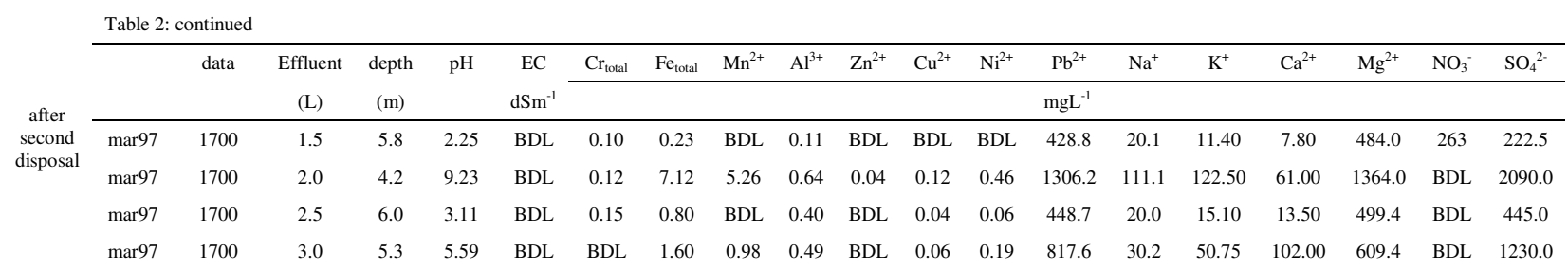

EC: electrical conductivity, BDL: below detection limit; n.a: not analysed

Table 3: Physical and chemical characteristics of reference soil

\begin{tabular}{|c|c|c|c|c|c|c|}
\hline \multirow{2}{*}{ Parameters } & \multicolumn{6}{|c|}{$\operatorname{depth}(\mathrm{m})$} \\
\hline & 0.5 & 1.0 & 1.5 & 2.0 & 2.5 & 3.0 \\
\hline $\mathrm{pH}$ & 4.2 & 4.2 & 4.1 & 4.2 & 4,1 & 4,1 \\
\hline Organic matter $(\%)$ & 1.0 & 1.0 & 0.7 & 0.7 & 0.7 & 0.7 \\
\hline \multicolumn{7}{|c|}{ Exchangeable $\left(\mathrm{mmol}_{\mathrm{c}} \mathrm{dm}^{-3}\right)$} \\
\hline $\mathrm{K}^{+}$ & 7 & 2 & 2 & 1 & 1 & 3 \\
\hline $\mathrm{Ca}^{2+}$ & 7 & 3 & 6 & 1 & 3 & 1 \\
\hline $\mathrm{Mg}^{2+}$ & 5 & 3 & 6 & 4 & 6 & 12 \\
\hline $\mathrm{H}+\mathrm{Al}$ & 28 & 34 & 28 & 34 & 47 & 52 \\
\hline $\mathrm{CEC}$ & 47 & 42 & 42 & 40 & 57 & 68 \\
\hline $\mathrm{SB}(\%)$ & 40 & 19 & 33 & 15 & 17 & 24 \\
\hline \multicolumn{7}{|c|}{ Texture $(\%)$} \\
\hline Clay & 25 & 26 & 24 & 24 & 22 & 12 \\
\hline Silt & 7 & 8 & 7 & 8 & 11 & 14 \\
\hline Sand & 68 & 65 & 67 & 66 & 67 & 74 \\
\hline \multicolumn{7}{|c|}{ Total (\%) } \\
\hline $\mathrm{Na}_{2} \mathrm{O}$ & 0.009 & BDL & $\mathrm{BDL}$ & BDL & BDL & 0.025 \\
\hline $\mathrm{K}_{2} \mathrm{O}$ & 0.530 & 0.510 & 0.450 & 0.450 & 0.740 & 1.240 \\
\hline $\mathrm{MgO}$ & 0.320 & 0.290 & 0.260 & 0.280 & 0.520 & 0.840 \\
\hline $\mathrm{CaO}$ & 0.056 & 0.036 & 0.032 & 0.021 & 0.018 & 0.030 \\
\hline $\mathrm{BaO}$ & 0.036 & 0.039 & 0.033 & 0.034 & 0.041 & 0.069 \\
\hline $\mathrm{P}_{2} \mathrm{O}_{5}$ & 0.064 & 0.058 & 0.050 & 0.053 & 0.069 & 0.081 \\
\hline $\mathrm{SO}_{3}$ & 0.047 & $\mathrm{BDL}$ & BDL & 0.005 & BDL & BDL \\
\hline $\mathrm{Cr}_{2} \mathrm{O}_{3}$ & 0.060 & 0.051 & 0.049 & 0.050 & 0.070 & 0.070 \\
\hline $\mathrm{MnO}$ & 0.060 & 0.059 & 0.057 & 0.061 & 0.062 & 0.074 \\
\hline $\mathrm{ZnO}$ & 0.011 & 0.008 & 0.007 & 0.005 & 0.005 & 0.005 \\
\hline $\mathrm{Fe}_{2} \mathrm{O}_{3}$ & 5.85 & 6.05 & 5.65 & 6.05 & 7.05 & 6.75 \\
\hline $\mathrm{Al}_{2} \mathrm{O}_{3}$ & 9.70 & 9.80 & 9.10 & 9.50 & 10.90 & 9.90 \\
\hline $\mathrm{SiO}_{2}$ & 79.60 & 79.40 & 80.70 & 79.80 & 76.60 & 77.40 \\
\hline $\mathrm{TiO}_{2}$ & 2.80 & 2.90 & 2.76 & 2.88 & 3.05 & 2.66 \\
\hline
\end{tabular}

BDL: below detection limit; CEC: cation exchange capacity (sum of $\mathrm{K}, \mathrm{Ca}, \mathrm{Mg}, \mathrm{H}+\mathrm{Al}$ ), $\mathrm{SB}$ : percent base saturation ((sum of $\mathrm{K}, \mathrm{Ca}, \mathrm{Mg} /$ CEC)*100)

The analysis shows that application of tannery effluents increases $\mathrm{Mn}^{2+}, \mathrm{Pb}^{2+}$ solubility and availability in soil solution.

Factor 2 accounted for $12.21 \%$ of the total variance related to $\mathrm{SO}_{4}{ }^{2-}$. It is likely that the availability of sulphate depends on higher concentrations in the effluents.

The third Factor with $10.16 \%$ of the total variance including $\mathrm{Zn}^{2+}$ and $\mathrm{Cu}^{2+}$, indicating a behavior similar in the soil, not correlated to soil solution $\mathrm{pH}$ (Table 4), possibly due to adsorption reactions with inorganic and organic colloids ${ }^{[42]}$.

Nitrate in high concentrations in soil solution is a contaminant that can leach and contribute to degrade groundwater quality. Therefore, $\mathrm{NO}_{3}^{-}$was only included at Factor 4 (8.49\%). Nitrification, a process that includes microbial activity, presents different dynamics than usual ionic processes and can explain this factor, representative of this delayed process.

The factor analysis using the three first Factors was suitable for explaining the variance of 12 of the 14 variables (Table 5) and it was shown to be an interesting tool to verify the results.

One concludes that high disposal caused significant alteration of soil solution and that clay content could enhance an accumulation of leached cationic species by clay surface adsorption at approximately $2.0 \mathrm{~m}$ depth. This depth showed the lower base saturation (Table 3), which contributes to cation exchange and enhances their accumulation. 
Am. J. Appl. Sci., 4 (12): 1063-1070, 2007

Table 4: Correlation coefficients of the 17 physico-chemical variables of soil solution. The symbol * show statistical significance at 0.01 level

\begin{tabular}{|c|c|c|c|c|c|c|c|c|c|c|c|c|c|c|c|c|c|}
\hline & $\mathrm{pH}$ & $\mathrm{EC}$ & $\mathrm{Cr}_{\text {total }}$ & $\mathrm{Fe}_{\text {total }}$ & $\mathrm{Mn}^{2+}$ & $\mathrm{Al}^{3+}$ & $\mathrm{Zn}^{2+}$ & $\mathrm{Cu}^{+}$ & $\mathrm{Ni}^{2+}$ & $\mathrm{Pb}^{2+}$ & $\mathrm{Na}^{+}$ & $\mathrm{K}^{+}$ & $\mathrm{Ca}^{2+}$ & $\mathrm{Mg}^{2+}$ & $\mathrm{NO}_{3}{ }^{-}$ & $\mathrm{SO}_{4}{ }^{2-}$ & $\mathrm{Cl}^{-}$ \\
\hline $\mathrm{pH}$ & 1 & & & & & & & & & & & & & & & & \\
\hline $\mathrm{EC}$ & $-0.70 *$ & 1 & & & & & & & & & & & & & & & \\
\hline $\mathrm{Cr}_{\text {total }}$ & -0.05 & 0.07 & 1 & & & & & & & & & & & & & & \\
\hline $\mathrm{Fe}_{\text {total }}$ & 0.16 & -0.10 & $0.30^{*}$ & 1 & & & & & & & & & & & & & \\
\hline $\mathrm{Mn}^{2+}$ & $-0.77^{*}$ & $0.79^{*}$ & 0.11 & 0.04 & 1 & & & & & & & & & & & & \\
\hline $\mathrm{Al}^{3+}$ & $-0.71 *$ & $0.69 *$ & 0.05 & -0.04 & $0.62 *$ & 1 & & & & & & & & & & & \\
\hline $\mathrm{Zn}^{2+}$ & -0.20 & $0.31^{*}$ & 0.22 & 0.10 & 0.41 & 0.34 & 1 & & & & & & & & & & \\
\hline $\mathrm{Cu}^{+}$ & -0.06 & 0.13 & -0.13 & -0.09 & 0.09 & 0.16 & $0.37 *$ & 1 & & & & & & & & & \\
\hline $\mathrm{Ni}^{2+}$ & $-0.64 *$ & $0.74 *$ & 0.13 & -0.03 & $0.76^{*}$ & $0.72^{*}$ & $0.40 *$ & 0.17 & 1 & & & & & & & & \\
\hline $\mathrm{Pb}^{2+}$ & $-0.48^{*}$ & $0.46^{*}$ & -0.08 & 0.06 & $0.53^{*}$ & $0.59^{*}$ & 0.27 & 0.10 & $0.69 *$ & 1 & & & & & & & \\
\hline $\mathrm{Na}^{+}$ & $-0.69 *$ & $0.83^{*}$ & 0.02 & -0.12 & $0.78^{*}$ & $0.63^{*}$ & $0.31^{*}$ & 0.10 & $0.79 *$ & $0.48^{*}$ & 1 & & & & & & \\
\hline $\mathrm{K}^{+}$ & $-0.29 *$ & $0.63^{*}$ & $-0.33^{*}$ & -0.07 & $0.49^{*}$ & $0.49^{*}$ & 0.14 & 0.13 & $0.62 *$ & $0.44 *$ & $0.59^{*}$ & 1 & & & & & \\
\hline $\mathrm{Ca}^{2+}$ & $-0.59 *$ & $0.78^{*}$ & 0.18 & 0.13 & $0.85^{*}$ & $0.63^{*}$ & $0.45^{*}$ & 0.07 & $0.79 *$ & $0.66^{*}$ & $0.71^{*}$ & $0.64^{*}$ & 1 & & & & \\
\hline $\mathrm{Mg}^{2+}$ & $-0.66^{*}$ & $0.80^{*}$ & 0.07 & 0.05 & $0.85^{*}$ & $0.62^{*}$ & $0.46^{*}$ & 0.13 & $0.79^{*}$ & $0.66^{*}$ & $0.72 *$ & $0.60 *$ & $0.93^{*}$ & 1 & & & \\
\hline $\mathrm{NO}_{3}^{-}$ & $-0.30^{*}$ & $0.47^{*}$ & 0.20 & 0.21 & $0.41^{*}$ & 0.15 & 0.11 & $-0.29 *$ & $0.28 *$ & 0.17 & $0.35^{*}$ & 0.24 & $0.43^{*}$ & $0.41^{*}$ & 1 & & \\
\hline $\mathrm{SO}_{4}{ }^{2-}$ & 0.06 & 0.11 & 0.13 & 0.05 & 0.08 & -0.10 & 0.20 & 0.01 & 0.05 & -0.06 & 0.22 & -0.04 & 0.05 & -0.04 & 0.00 & 1 & \\
\hline $\mathrm{Cl}^{-}$ & $-0.61 *$ & $0.83^{*}$ & 0.09 & 0.08 & $0.75^{*}$ & $0.66^{*}$ & $0.29 *$ & 0.08 & $0.79^{*}$ & $0.51 *$ & $0.78^{*}$ & $0.71 *$ & $0.80^{*}$ & $0.81^{*}$ & $0.41^{*}$ & 0.01 & 1 \\
\hline
\end{tabular}

EC: electrical conductivity

Table 5: Varimax rotated factor matrix for 14 physico-chemical variables $^{\mathrm{a}}$

\begin{tabular}{lrrrr}
\hline Variables & Factor 1 & Factor 2 & Factor 3 & Factor 4 \\
\hline $\mathrm{pH}$ & -0.685 & 0.282 & 0.038 & -0.380 \\
$\mathrm{EC}$ & 0.701 & -0.302 & -0.037 & 0.519 \\
$\mathrm{Fe}_{\text {total }}$ & -0.169 & 0.594 & 0.000 & -0.008 \\
$\mathrm{Mn}^{2+}$ & 0.888 & -0.036 & -0.012 & 0.160 \\
$\mathrm{Zn}^{2+}$ & 0.144 & 0.183 & 0.849 & 0.182 \\
$\mathrm{Cu}^{2+}$ & -0.131 & -0.117 & 0.874 & -0.150 \\
$\mathrm{~Pb}^{2+}$ & 0.763 & -0.198 & -0.007 & 0.125 \\
$\mathrm{Na}^{+}$ & 0.849 & -0.004 & 0.011 & 0.433 \\
$\mathrm{~K}^{+}$ & 0.785 & 0.493 & -0.020 & -0.127 \\
$\mathrm{Ca}^{2+}$ & 0.867 & 0.201 & 0.023 & 0.181 \\
$\mathrm{Mg}^{2+}$ & 0.934 & 0.052 & 0.015 & 0.023 \\
$\mathrm{NO}_{3}^{-}$ & 0.129 & 0.193 & 0.028 & 0.920 \\
$\mathrm{SO}_{4}^{-}$ & 0.279 & 0.832 & 0.064 & 0.186 \\
$\mathrm{Cl}^{-}$ & 0.927 & 0.145 & 0.039 & -0.045 \\
Eingenvalue & 6.77 & 1.71 & 1.42 & 1.19 \\
$\%$ Variance explained & 48.35 & 12.21 & 10.16 & 8.49 \\
$\%$ cumulative variance & 48.35 & 60.56 & 70.71 & 79.20 \\
\hline${ }^{2}$ marked loadings are $>0.7$, EC: electrical conductivity.
\end{tabular}

The higher decrease of clay contents and the increase of exchangeable- $\mathrm{Mg}^{2+}$ at $3.0 \mathrm{~m}$ depth suggest the occurrence of saprolite. Therefore, the cation exchange capability at this depth does not represent the real soil exchange capacity.

\section{CONCLUSION}

The elaboration of data indicated that the impact of tannery effluents on acid soils is notable because of a general increase of heavy metals availability, with the exception of total $\mathrm{Cr}$ and $\mathrm{Fe}$, due to the change in soil $\mathrm{pH}$ after disposal.

The factor analysis allowed selecting four factors: salinity, $\mathrm{SO}_{4}{ }^{2-}, \mathrm{Zn}^{2+}$ and $\mathrm{Cu}^{2+}$ and $\mathrm{NO}_{3}{ }^{-}$. It is relevant to consider also the active role played by organic matter in the soil and active biotic components at different depths, the latter being directly involved in some enzymatic soil processes such as oxidation/reduction and nitrification activity.

We must take into account that $\mathrm{Mn}^{2+}$ was released in soil solution related to $\mathrm{Mn}$-oxide reduction caused by $\mathrm{Cr}$ (III) input and $\mathrm{pH}$ decrease. On the other hand, chromium, the main heavy metal in the effluents, was not detected in the available forms, probably due to coprecipitation reactions of $\mathrm{Cr}$ and $\mathrm{Fe}$ and to sorption onto oxides, oxi-hydroxides and hydroxides. It is remarkable the importance of these mineral soil constituents for a reduction of $\mathrm{Cr}$ availability and downward migration in the soil profile.

\section{ACKNOWLEDGEMENTS}

To "Fundação de Amparo à Pesquisa do Estado de São Paulo" (FAPESP) and to the tannery factory "Curtume Monte Aprazível" for financial aid. To "Conselho Nacional de Pesquisa Científica e Tecnológica" (CNPq) for the Grant. 


\section{REFERENCES}

1. Facchinelli, A., E. Sacchi and L. Mallen, 2003. Multivariate statistical and GIS-based approach to identify heavy metal sources in soils. Environ. Poll., 114: 313-324.

2. Kumru, M.N. and M. Bakaç, 2003. R-mode factor analysis applied to the distribution of elements in soils from the Aydin basin, Turkey. J. Geochem. Explor., 77: 81-91.

3. Rémy, S., P. Prudent, C. Hissler, J.L. Probst and G. Krempp, 2003. Total mercury concentrations in an industrialized catchments, the Thur River basin (north-eastern France): Geochemical background level and contamination factors. Chemosphere, 52: 635-644.

4. Khan, Z. and Y. Anjaneyulub, 2005. Influence of soil components on adsorption-desorption of hazardous organics-development of low cost technology for reclamation of hazardous waste dumpsites. J. Hazard. Mater., B118: 161-169.

5. Subbarao, C., N.V. Subarao and S.N. Chandu, 1996. Characterization of groundwater contamination using factor analysis. Environ. Geol., 28: 175-180.

6. Tariq, S.R., M.H. Shah, N. Shaheen, A. Khalique, S. Manzoor and M. Jaffar, 2005. Multivariate analysis of trace metal levels in tannery effluents in relation to soil and water: A case study from Peshawar, Pakistan. J. Environ. Manag., pp: 1-10.

7. Claas, J.C., 1994. R. A. Maia. Manual básico de resíduos de curtumes. SENAI-PADCT-CNPq, Porto Alegre.

8. Instituto Brasileiro de Geografia e Estatística.http//www.ibge.gov.br

9. Oliveira, J.B., M.N. Camargo, M. Rossi and B.C. Filho, 1999. Mapa pedológico do estado de São Paulo. Instituto Agronômico de Campinas, Embrapa Solos, Rio de Janeiro.

10. Tessier, A., P.G.C. Campbell and M. Bisson, 1979. Sequential extraction procedure for the speciation of particulate trace metal. Anal. Chem., 51: 844851.

11. Beckett, P.H.T., 1989.The use of extractants in studies on trace metals in soils, sewage sludge and sludge-treated soils. Adv. Soil Sci., 9: 143-176.

12. McBride, M.B. 1989. Reactions controlling heavy metal solubility in soils. Adv. Soil Sci., 10: 1-56.

13. Puls, R.W., C.J. Paul and D.A. Clark, 1992. Distribution, speciation and transformation of chromium in contaminated soils and aquifer sediments. Soil Sci. Soc. Am. J., pp: 455-457.
14. Balasoiu, C.F., G.J. Zagury and L. Deschênes, 2001. Partitioning and speciation of chromium, copper and arsenic in CCA-contaminated soils: influence of soil composition. Sci. Total Environ., 280: 239-255.

15. Nriagu, J., S. Beaubien and D. Blowes, 1993. Chemistry of chromium in lakes. Environ. Rev., 1: 104-120.

16. Eary, L.E. and D. Rai, 1989. Kinetics of chromate reduction by ferrous ions derived from hematite and biotite at $25^{\circ} \mathrm{C}$. Am. J. Sci., 289: 180-213.

17. Eary, L.E. and D. Rai, 1991. Chromate reduction by subsurface soils under acidic conditions. Soil Sci. Soc. Am. J., 55: 676-683.

18. Griffin, R.A., A.K. Au and R.R. Frost, 1977. Effect of $\mathrm{pH}$ on adsorption of chromium from landfillleachate by clay mineral. J. Environ. Sci. Health, A12: 431 - 449 .

19. Charlet, L. and A.A. Manceau, 1992. X-Ray absorption spectroscopic study of the sorption of $\mathrm{Cr}$ (III) at the oxide-water interface. J. Colloid \& Interface Sci., 148: 443-458.

20. Nikolaidis, N.P., G.A. Robbins, M. Scherer, B. McAninch, G. Binkhorst, J. Asikainesn and S.L. Suib, 1994. Vertical distribution and partitioning of chromium in a glaciofluvial aquifer. Ground Water Monitoring \& Remediation, Summer, pp: 150-159.

21. Bartlett, R.J. and B.R. James, 1979. Behavior of chromium in soils: III. Oxidation. J. Environ. Qual., 8: 31-35.

22. Fendorf, S.E., 1995. Surface reactions of chromium in soils and waters. Geoderma, 67: 55-71.

23. Kim, J.G., J.B. Dixon, C.C. Chusuei and Y. Deng, 2002. Oxidation of chromium(III) to (VI) by manganese oxides. Soil Sci. Soc. Am. J., 66: 305315.

24. Stepniewska, Z., K. Bucior and R. P. Bennicelli, 2004. The effects of $\mathrm{MnO}_{2}$ on sorption and oxidation of $\mathrm{Cr}(\mathrm{III})$ by soils. Geoderma, 122: 291296.

25. Tzou, Y.M., R.H. Loeppert and M.K. Wang, 2002. Effect of organic complexing ligands on $\mathrm{Cr}$ (III) oxidation by MnOx. Soil Sci., 167: 729-738.

26. Davidson, J.M., P.S.C. Rao and P. Nkedi-Kizza, 1983. Physical processes influencing water and solute transport in soils. in: D. W. Nelson, D. E. Elrick, K. K. Tanji (Eds.), Chemical mobility and reactivity in soil-systems. Soil Sci. Soc. Am. J., pecial publication, 11: 262.

27. Thomasson, M.J. and P.J. Wierenga, 2003. Spatial variability of the effective retardation factor in an unsaturated field soil. J. Hydrol., 272: 213-225. 
28. Vogel, H.J. and K. Roth, 2003. Moving through scales of flow and transport in soil. J. Hydrol., 272: 95-106.

29. Simeonov, V., J.A. Stratis, C. Samara, G. Zahcariadis, D. Voutsa, A. Anthemidis, M. Sofoniou and T. Kouimtzis, 2003. Assessment of the surface water quality in Northern Greece. Water Res., 37: 4119-4124.

30. Lambrakis, N., A. Antonakos and G. Panagopoulos, 2004. The use of multicomponent statistical analysis in hydrogeological environmental research. Water Res., 38: 18621872.

31. Shukla, M.K., R. Lal and M. Ebinger 2006. Determining soil quality indicators by factor analysis. Soil Tillage Res., 87: 194-204.

32. American Society for Testing and Materials, 1996. Standards on ground water and vadose zone investigations: drilling, sampling, well installation and abandonment procedures. ASTM Committee D-18 on Soil e Rock. Publication Code Number (PCN): 03-418196-38.

33. APHA, AWWA, WEF, 1995. Standard methods for the examination of water and wastewater. 19th ed., American Public Health Association. Washington, DC.

34. Van Raij, B., J.C. Andrade, H. Cantarella and J.A. Quaggio, 2001. Análise química para avaliação da fertilidade de solos tropicais. Instituto Agronômico, Campinas, SP.
35. Embrapa, 1997. Manual de métodos de análise de solo. Centro Nacional de Pesquisa de Solos, Rio de Janeiro.

36. Davis, J.C., 1986. Statistics and Data Analysis in Geology. New York, John Wiley \& Sons.

37. StatSoft, Inc. STATISTICA for Windows v.5.5 [Computer program manual], 1999. Tulsa.

38. Landon, M.K., G.N. Delin, S.C. Komor and C.P. Regan, 1994. Comparison of the stable-isotopic composition of soil water collected from suction lysimeters, wick samplers and cores in a sandy unsaturated zone. J. Hydrol., 224: 45-54.

39. Suarez, D.L, 1986. A soil water extractor that minimizes $\mathrm{CO}_{2}$ degassing and $\mathrm{pH}$ errors. Water Resource Res., 22: 876-880.

40. McBride, M.B., 1994. Environmental chemistry of soils. Oxford University Press. New York.

41. Surita, C.A., 1999. Avaliação de contaminação de águas intersticiais e solo devido a disposição de efluentes de curtimento em superfície. Um estudo em campo. Tese de Doutorado. Universidade de São Paulo.

42. Jeffery, J.J. and N.C. Uren, 1983. Copper and Zinc Species in the soil solution and the effects of soil pH. Aus. J. Soil Res., 21: 479-488. 